\title{
Numerical Modeling and Sensitivity Analysis on Microwave Heating of Plastic on a Large Scale
}

\author{
Sangjun Jeon', Jaekyung Kim ${ }^{2}$ and Daejong Yang ${ }^{1,3, *}$ \\ 1 Department of Future Convergence Engineering, Kongju National University, Cheonan 31080, Republic of \\ Korea; A20200301@smail.kongju.ac.kr \\ 2 Industrial Technology Research Institute, Kongju National University, Cheonan 31080, Republic of Korea; \\ kjk8431@gmail.com \\ 3 Department of Mechanical and Automotive Engineering, Kongju National University, Cheonan 31080, Re- \\ public of Korea \\ * Correspondence: daejong@kongju.ac.kr
}

\begin{abstract}
To reduce the carbon emissions during heating in the manufacturing process, microwaves have attracted significant attention. Microwave has a lot of advantages rather than traditional heating method such as rapid heating, lower thermal damage and eco-friendly process. In order to apply microwaves to manufacturing process, uniform and efficient heating is required. We have analyzed the effect of various design parameters such as cavity heights, the application of the reflector, and the number and positions of waveguides for uniform and efficient heating by numerical simulation and verified that by experiment. The results showed that a slight change in the cavity height altered the electromagnetic field distribution and heating parameters, such as the coefficient of variance and power absorption efficiency. With reflectors installed, uniform heating was achieved and power absorption was improved, with the spherical reflector showing the maximum efficiency. The use of double waveguides heated the target material in a uniform manner. An increase in the power supply also led to uniform heating. This large-scale analysis will be helpful in applying microwaves to actual industrial sites.
\end{abstract}

Keywords: microwave heating; cavity; reflector; double-waveguide; uniform heating; high heating efficiency

\section{Introduction}

Fossil fuels represent approximately $80 \%$ of the total energy sources as of 2020 [1]. Not only energy source but also chemicals produced through fossil fuels are used in our daily lives as well as in various fields, such as petroleum, construction, and electricity [2]. However, the processes of manufacturing or using such energy and chemical products emit a large amount of carbon dioxide $\left(\mathrm{CO}_{2}\right)$. This leads to an annual emission of $33.5 \mathrm{Gt}$ of $\mathrm{CO}_{2}$, which gas acts as a greenhouse gas. $\mathrm{CO}_{2}$ emissions into the atmosphere cause global warming by rapidly increasing the temperature of the earth, and there have been active discussions among countries around the world to reduce the use of fossil fuels. To manage $\mathrm{CO}_{2}$ emissions caused by fossil fuels, clean renewable energy sources, such as sunlight and wind power, have been used instead of conventional fossil fuels or low-carbon methods that reduce $\mathrm{CO}_{2}$ emissions in the process of converting fossil fuels into different energy sources, such as electricity. Although it is getting better in terms of energy production in continuous efforts to reduce carbon emissions, the consumption of plastic products produced using fossil fuels is rapidly increasing each year, retrogressing the process of low-carbon transitions. Therefore, the need to reduce a large amount of $\mathrm{CO}_{2}$ in the process of producing plastics has emerged, and leading countries have announced carbon reduction policies that mainly target carbon emission reduction during plastic production to address this problem. Researchers have focused on various methods to reduce carbon 
emissions in various processes, such as sintering, drying, and synthesis. Among them, processing materials using microwaves have attracted significant attention [3].

The traditional heating method uses heat transfer involves heat radiation, convection, and conduction. This method requires heating for several hours to reach the target temperature due to the heat transfer from the outside heat source to the inside; the high energy consumption and long-term exposure to high temperatures lead to surface damage and property deterioration [4]. In contrast, in microwave heating, heating is performed by directly vibrating molecules or through particle charge [5]. Thus, microwave heating leads to lower surface damage in materials and has a shorter heating time than traditional heating [6]; furthermore, it is eco-friendly because it consumes less energy and emits lower amounts of carbon than conventional heating. According to Gupta [7], carbon emissions can be reduced by several dozen times compared to the traditional heating process if materials are heated using microwaves. Due to this advantage, various studies have focused on heating using microwaves. For example, carbon nanotubes and hydrogen with higher qualities were extracted by recycling plastics using microwaves, and the process was also simpler than the conventional method [8]. Suriapparao [9] produced bio diesel through microwave pyrolysis after mixing biomass with plastics and emphasized that the pyrolysis process that uses microwaves is an excellent means to obtain fuel in an ecofriendly manner. In addition, researches have been conducted for various processes, such as sintering, synthesis, and drying, using microwaves instead of the traditional heating process, and they were also reported that microwaves are eco-friendly and more efficient than the conventional heating method [10-12].

Despite these advantages, microwaves have the shortcoming of non-uniform heating. In the traditional heating process, the temperature difference between the surface and inside of the material is a problem. In the microwave heating process, however, hot spots with high temperature in specific areas may occur as a non-uniform electromagnetic field distribution is caused by the intrinsic characteristics of microwaves. Hot spots degrade the quality of the target material and may burn or explode the material in severe cases [13]. Numerous researchers have attempted to address this problem and have proposed two main approaches to improve the uniformity of heating. The first method is changing the geometry of the cavity or moving the target material so that the material can be exposed to a uniform electromagnetic field, and the other method is irradiating microwaves with a wavelength suitable for the target material [14].

Yi [15] installed a mode stirrer and a conveyor belt that influence the heating effect inside the cavity to uniformly irradiate microwaves, and He [16] increased the heating efficiency and uniformity by applying a rotary radiation structure. Tang [17] reported that the microwave frequency had a significant impact on the heating effect. In addition, various studies have been conducted on the heating uniformity and heating effect suitable for material processing conditions by applying various methods inside the cavity such as installed muffle and multiple waveguides [18-20]. Despite such studies, the application of microwaves in industries is challenging. Most experiments have been performed on a laboratory scale, and few studies that are applicable to large-capacity equipment have been conducted. Therefore, in this study, we present the pilot-scale applicability of microwaves heating by conducting a real-scale experiment and numerical analysis.

In this study, the hopper model, one of the large-capacity equipment models applicable to actual industrial sites, was designed, and results of numerical analysis were compared with the experimental results. Various cavity heights, the application of the reflector, and the number and positions of waveguides were considered. The electromagnetic field distribution and heating effect according to the design parameters were calculated through simulation to analyze the influence of each parameter.

\section{Simulation and Experiments}

\subsection{Numerical Model}


To process a material using microwaves, it is essential to predict the temperature profile of the target material [21]. It is difficult, however, to visually examine the temperature profile and electromagnetic field distribution inside a closed metal cavity during an actual test. Therefore, the temperature profile and electromagnetic field distribution were predicted through computer-based finite element analysis. Commercial finite element analysis software (COMSOL Multiphysics ${ }^{\circledR}$ V5.6, COMSOL Inc, Sweden) widely used for microwave heating analysis was used for numerical simulation. Simulation was conducted under the frequency-transient condition using the heat transfer, electromagnetic, and transport diluted species modules. Figure 1 shows the relationship and interaction among the modules. The microwaves generated in the electromagnetic module form an electromagnetic field distribution inside the cavity based on Maxwell's equation. The heat transfer module is associated with the electromagnetic module, and the electromagnetic field loss is regarded as a heat source in the heat transfer part. Based on the calculated heat source, the temperature and moisture distribution of the target material were derived in the heat transfer and transport diluted species modules. All these processes are accompanied by the energy-mass conservation law.

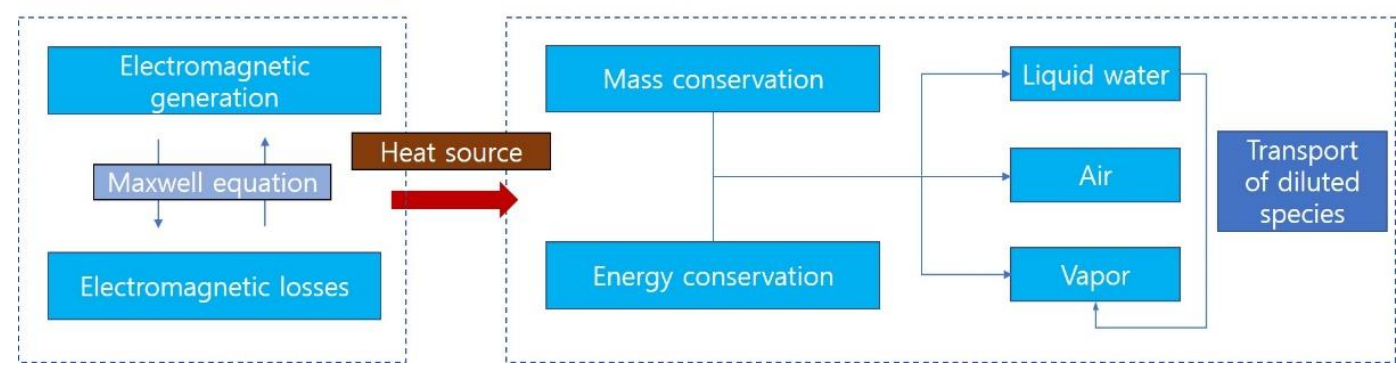

Figure 1. Simulation relationship between electromagnetic, heat transfer in porous and transport diluted species module.

\subsubsection{Geometry}

Figure 2a shows the cavity model with the hopper structure to be used in the experiment and analysis. $2.45 \mathrm{GHz}$ magnetron was attached to the cavity side face, which operated in the transverse electric 10 mode (TE10). Microwaves with a power of $1 \mathrm{~kW}$ were generated by the magnetron and propagated into the cavity through rectangular waveguides. The attached waveguides had dimensions of WR-340 (a width of $86.4 \mathrm{~mm}$ and a height of $43.2 \mathrm{~mm}$ ), and simulation was performed while their positions and number were varied. The dimensions of the cavity and the design parameters to be applied to simulation are summarized in Figure 2a,b. The cavity was cylindrical and had a diameter and height of 430 and $461 \mathrm{~mm}$, respectively. The lower hopper had a height of $272 \mathrm{~mm}$, an upper diameter of $430 \mathrm{~mm}$, and a lower diameter of $58 \mathrm{~mm}$. The height of the cavity was adjusted based on the wavelength of the $2.45 \mathrm{GHz}$ microwave. The cavity walls and waveguides were made of copper, which reflects microwaves. In addition, $25 \mathrm{~kg}$ polycarbonate was placed inside the hopper. Table 1 summarizes the material properties of the cavity and the target material required for analysis. 
(a)

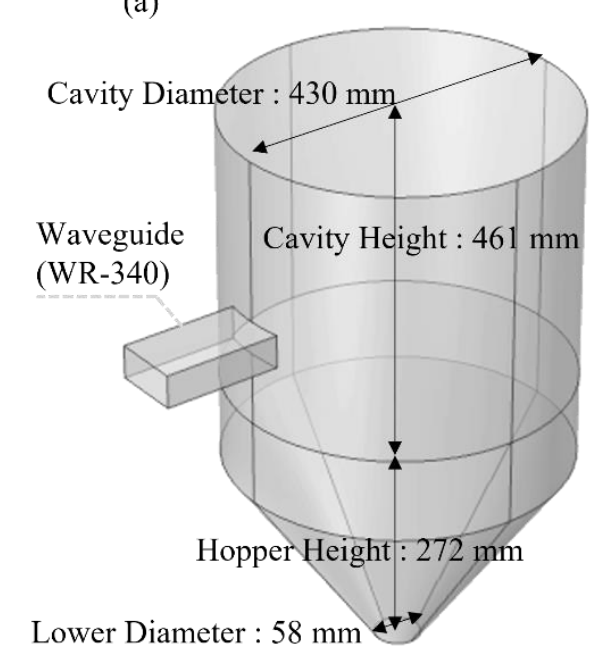

(b)

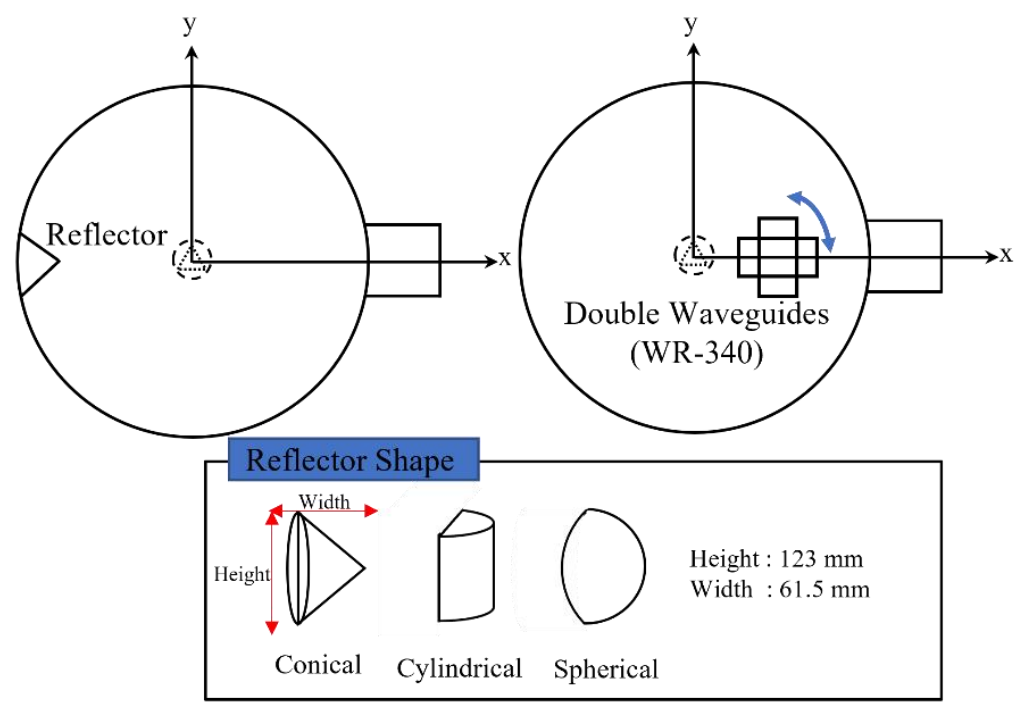

Figure 2. (a) Geometry of microwave hopper structure and (b) design parameter of simulation for microwave heating simulation.

\subsubsection{Assumption}

The following assumptions were applied to the analysis model to efficiently simulate complex phenomena during microwave heating [22].

1. The cavity walls and waveguides are considered to be perfectly conducting.

2. The cavity walls and waveguides are completely insulated.

3. All materials are homogeneous.

4. Chemical reactions are not considered in the heating process.

5. The heat transfer model is applied only to polycarbonate.

6. Shrinkage of pellets is not considered.

7. The influence of air flow is not considered.

\subsubsection{Governing Equations} Eq. [23].

In this simulation, the governing equation of electric field wave is given by following

$$
\nabla \times \mu^{-1} \cdot \nabla \boldsymbol{E}-k_{0}\left(\varepsilon_{0} \varepsilon_{r}-\frac{j \sigma}{\omega}\right) \boldsymbol{E}=0
$$

Where $E$ is Electric field vector, $\omega$ is angular frequency, $\sigma$ is the conductivity, $\varepsilon_{0}\left(8.854 \times 10^{-12} \mathrm{~F} / \mathrm{m}\right)$ is permittivity of free space and $k_{0}$ is the wave number of free space, which is defined as:

$$
k_{0}=\omega / c_{0}
$$

Where $c_{0}$ is the speed of light in vacuum.

The relative permeability of $\mu_{r}$ can be define as[24]:

$$
\mu_{r}=\mu^{\prime}-\mu^{\prime \prime}
$$

Where $\mu^{\prime}$ is the magnetic constant that is a measure of the ability of the dielectric materials to store magnetic energy, $\mu^{\prime \prime}$ represents the loss of magnetic field energy.

The relative permittivity of $\varepsilon_{r}$ can be defines as [25]:

$$
\varepsilon_{r}=\varepsilon^{\prime}-j \varepsilon^{\prime \prime}
$$

Where $\varepsilon^{\prime}$ is the dielectric constant that represents ability to store electromagnetic energy, $\varepsilon^{\prime \prime}$ is the loss factor that is known as dissipate the absorbed electromagnetic energy, converting electromagnetic energy into heat.

When electromagnetic waves interact with material, electromagnetic wave energy losses that converted into heat based on Fourier's energy balance equation within the area. The heat transfer in hopper model can be expressed by Eq. (5). 


$$
\rho C_{p} \boldsymbol{u} \cdot \nabla T+\nabla \cdot \boldsymbol{q}=Q_{e}
$$

Where $\rho$ is the density, $C_{p}$ is the specific heat capacity, $\boldsymbol{u}$ is the velocity field, $T$ is the temperature. Heat flux $\mathrm{q}$ can be define as:

$$
\boldsymbol{q}=-k \nabla T
$$

Where $\mathrm{k}$ is the thermal conductivity.

The electromagnetic losses, $Q_{e}$ is regarded as heat source place right side of Eq. (7) $Q_{e}$ can be defined as

$$
Q_{e}=Q_{r h}+Q_{m l}
$$

Where the resistive losses are

$$
Q_{r h}=\frac{1}{2} \operatorname{Re}\left(\boldsymbol{J} \cdot \boldsymbol{E}^{*}\right)
$$

And the magnetic losses are

$$
Q_{m l}=\frac{1}{2} R e\left(i \omega \boldsymbol{B} \cdot \boldsymbol{H}^{*}\right)
$$

\begin{tabular}{|c|c|c|c|}
\hline No. & Parameters & Value or Expression & Reference \\
\hline 1 & Microwave frequency & $2.45 \mathrm{GHz}$ & - \\
\hline 2 & Microwave power & $1 \mathrm{~kW}$ & - \\
\hline 3 & $\begin{array}{l}\text { Air moisture concentra- } \\
\text { tion }\end{array}$ & $0.05 \mathrm{~mol} / \mathrm{m}^{3}$ & 4 \\
\hline 4 & $\begin{array}{l}\text { Dielectric constant of } \\
\text { polycarbonate }\end{array}$ & 2.35 & 8 \\
\hline 5 & $\begin{array}{l}\text { Loss factor of polycar- } \\
\text { bonate }\end{array}$ & $2 \times 10^{-4}$ & 8 \\
\hline 6 & Thermal conductivity & $\begin{array}{l}0.07182401 \mathrm{~W} /(\mathrm{m} \cdot \mathrm{K})+7.942708 \times 10^{-4} \mathrm{~W} /\left(\mathrm{m} \cdot \mathrm{K}^{2}\right) \cdot \mathrm{T} \\
-1.8525 \times 10^{-6} \mathrm{~W} /\left(\mathrm{m} \cdot \mathrm{K}^{3}\right) \cdot \mathrm{T}^{2}+2.126779 \times 10^{-9} \cdot \mathrm{W} /\left(\mathrm{m} \cdot K^{4}\right) \cdot \mathrm{T}^{3}\end{array}$ & 1 \\
\hline 7 & Polycarbonate density & $1050 \mathrm{~kg} / \mathrm{m}^{3}$ & 1 \\
\hline
\end{tabular}

Where $\boldsymbol{J}$ is the current density, $\boldsymbol{B}$ is the magnetic flux density, and $\boldsymbol{H}$ is the magnetic field intensity.

Table 1. Physical properties of polycarbonate

${ }^{1}$ is Comsol Built-in [23]

The existence of moisture important role in heating. Water evaporation and heat convection could affect temperature. When the temperature reached a certain level, the phase and distribution of moisture will be changed, which could influence the heating effect. Mass transfer process should be considered. The mass transfer solved in polycarbonate expressed as

$$
\nabla \cdot J_{i}+\mathrm{u} \cdot \nabla c_{i}=R_{i}
$$

Where $c_{i}$ denotes the concentration of species, $\mathrm{u}$ is the fluid velocity, $R_{i}$ is the molar production constant, $J_{i}$ is the diffusive flux vector, given as

$$
J_{i}=-D_{i} \cdot \nabla c_{i}
$$

Where $D_{i}$ is the diffusion coefficient.

\subsubsection{Boundary Condition}

(1) Electromagnetic Boundary Conditions

Cavity wall and waveguides are applied impedance boundary condition where the electromagnetic field is known to penetrate only a short distance outside the boundary [26]: 


$$
\begin{aligned}
\sqrt{\frac{\mu_{0} \mu_{r}}{\varepsilon_{0} \varepsilon_{r}-j \sigma_{e} / \omega}} & \boldsymbol{n} \\
& \times \boldsymbol{H}+\boldsymbol{E}-(\boldsymbol{n} \cdot \boldsymbol{E}) \\
& =\left(\boldsymbol{n} \cdot \boldsymbol{E}_{s}\right) \boldsymbol{n}-\boldsymbol{E}_{\boldsymbol{s}}
\end{aligned}
$$

where $\boldsymbol{E}_{\boldsymbol{s}}$ is the source electric field and $\boldsymbol{n}$ is the normal vector toward the exterior of the target boundary.

In this study, the rectangular waveguides are excited frequency of $2.45 \mathrm{GHz}$ that operate in TE10 mode.

The port boundary conditions are expressed as

$$
\beta=\frac{2 \pi}{c_{0}} \sqrt{v^{2}-v_{c}^{2}}
$$

where $\beta$ is the propagation constant, $v$ is the frequency of microwave, $v_{c}$ is the cutoff frequency.

(2) Thermal Condition

The thermal insulation boundary condition means that there is no heat flux, which is can be expressed by the following equation:

$$
-\boldsymbol{n} \cdot(-k \nabla T)=0
$$

(3) Mass Condition

Convection is applied to the target and can be expressed as

$$
\begin{gathered}
-\boldsymbol{n} \cdot(-D \nabla c)=J_{0} \\
J_{0}=k\left(c_{b}-c\right)
\end{gathered}
$$

Where $\mathrm{D}$ is the moisture diffusion coefficient in the sample, $c_{b}$ is the air moisture concentration, $k$ refers to the mass transfer coefficient, $J_{0}$ is the bottom face of sample and defined as no flux boundary condition.

\subsubsection{Mesh}

The mesh size is a very important element in simulation as it affects the convergence and accuracy of analysis. For example, when the mesh size decreases by half, the computation time increases by 16 times and the memory usage increases by 8 times [27]. According to the results of a previous study on numerical simulations, at least five mesh elements per wavelength are recommended for the proper mesh size in microwave heating [28]. The mesh element quality (MEQ), one of the most commonly used methods in previous studies, was evaluated by applying five or more mesh elements per wavelength for accurate simulation. In COMSOL Multiphysics, MEQ can be calculated using Eq. [29].

$$
M E Q=\frac{4 \sqrt{3} A}{h_{1}^{2}+h_{2}^{2}+h_{3}^{2}}
$$

Where $A$ is area and $h_{1}, h_{2}$ and $h_{3}$ are the side length of the triangle. 227146 meshes were used. As shown in Figure 3, the average mesh quality was 0.9, which exceeded the criterion (0.65) of previous studies. 
(a)

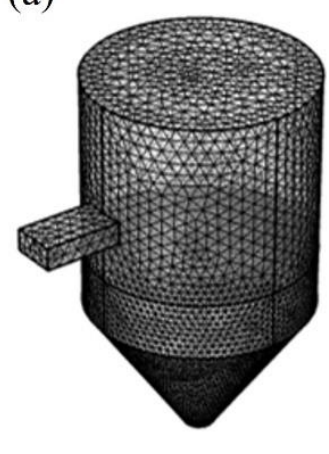

(b)

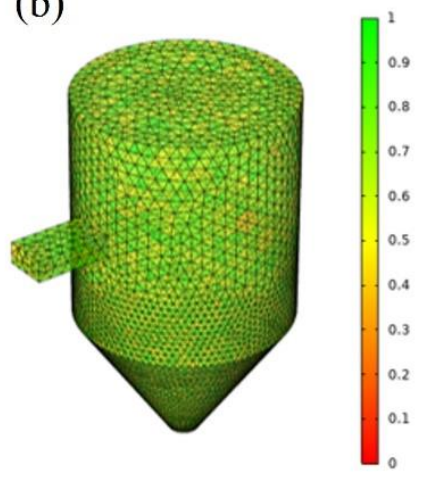

Figure 3. (a) Mesh element and (b) mesh quality evaluation of model.

\subsection{Experiments}

\subsubsection{Experimental Setup}

Figure 4a shows the experimental setup, and its specifications are the same as those mentioned in the simulation. As shown in Figure $4 \mathrm{~b}$, only one $1 \mathrm{~kW}$ magnetron was attached to the $221 \mathrm{~mm}$ position on side, or another magnetron was attached to the upper part at a position $120 \mathrm{~mm}$ away from the center. To measure the temperature, three thermocouples were inserted at depths of 197,106 , and $17 \mathrm{~mm}$ at $25^{\circ}$ intervals at the hopper height of $272 \mathrm{~mm}$.

(a)

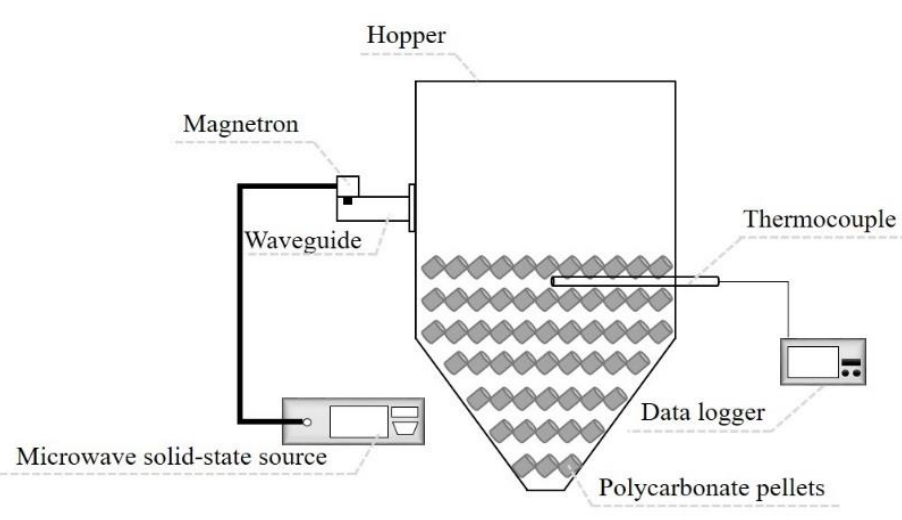

(b)

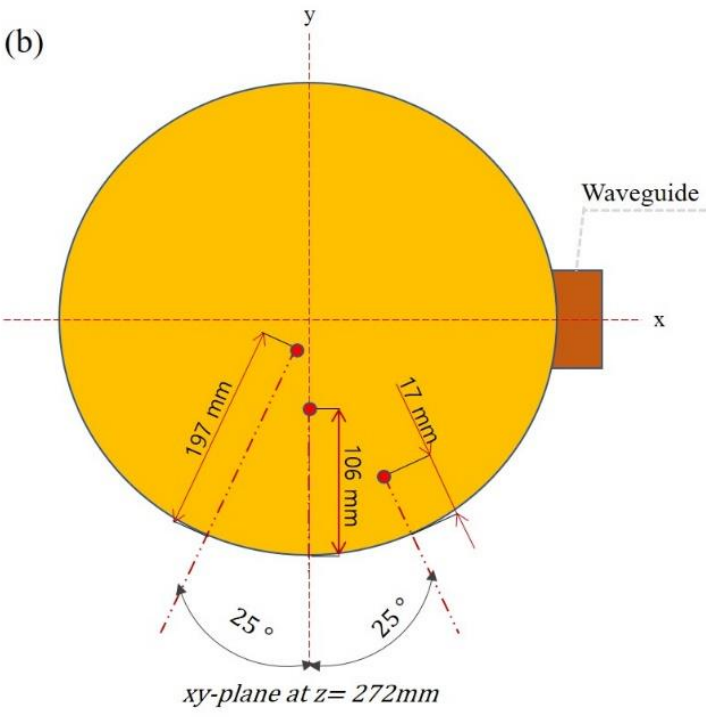

Figure 4. (a) Schematic illustration of experimental setup for microwave heating and (b) temperature sampling points.

\subsubsection{Experiments Procedure}

Polycarbonate was exposed to a humid environment for several hours to create conditions similar to actual industrial environments. To prevent the difference in heating effect caused by the moisture content difference, the moisture content was maintained at $0.11 \%$ by monitoring it using a moisture meter (MS-70, AND, Japan). After inserting $25 \mathrm{~kg}$ of polycarbonate pellets into the experimental setup, the magnetron was operated, and microwaves were irradiated for $600 \mathrm{sec}$. The temperature of the polycarbonate was measured every 2 sec using a thermocouple.

\subsubsection{Experiments Validate}

To verify the validity of the simulation model, the results of the experiment described above were compared with the simulation results. The atmospheric temperature at the 
time of the experiment, $25^{\circ} \mathrm{C}$, was set as the initial value, and the temperature values at the same positions as in the experimental setup were collected while $1 \mathrm{~kW}$ and $2 \mathrm{~kW}$ microwaves were irradiated inside the cavity for $600 \mathrm{sec}$. As shown in Figure 5, the root mean square error between the experimental values and simulation results was $1.2{ }^{\circ} \mathrm{C}$ when heating was performed using $1 \mathrm{~kW}$ for $10 \mathrm{~min}$ and $1.9{ }^{\circ} \mathrm{C}$ when $2 \mathrm{~kW}$ was supplied by installing two waveguides. The small errors between the simulation and experimental results confirmed that the numerical analysis model is reliable.

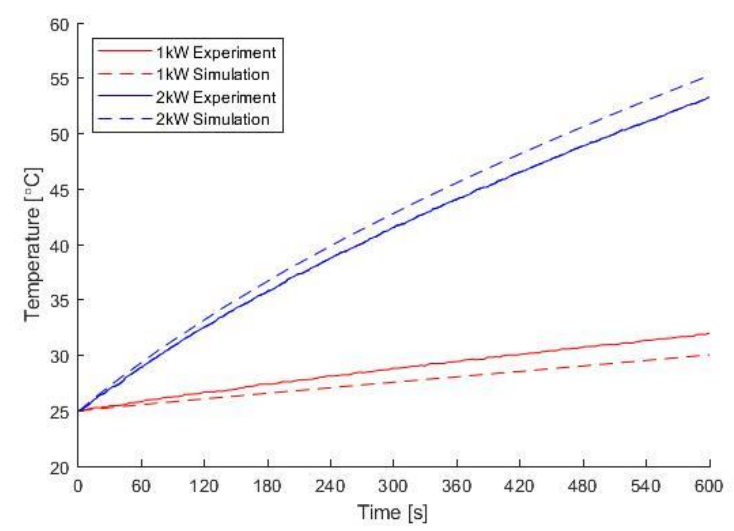

Figure 5. Temperature comparison between numerical simulation and experiment under $1 \mathrm{~kW}$ and $2 \mathrm{~kW}$ heating condition.

\section{Results and Discussion}

Microwaves are generated from a magnetron and are transmitted to the microwave cavity through the waveguide. In this instance, various design parameters, such as the cavity height, the geometry of the reflector inside the cavity, and the positions and number of microwave ports, reflect the microwaves in different directions, creating various electromagnetic field distributions. The created electromagnetic field converts the energy of the microwaves into thermal energy through interaction with objects, thereby causing different heating results. As mentioned in the introduction section, reducing hot spots through uniform heating is an important element for microwave-based heating. The following coefficient of variance (COV) has been widely used to quantify the heating uniformity after microwave irradiation [30].

$$
\begin{gathered}
\operatorname{COV}=\frac{1}{E(T)} \sqrt{\frac{1}{N} \sum_{1}^{N}\left(T_{i}-T_{0}\right)^{2}} \\
E(T)=T_{a}-T_{0}
\end{gathered}
$$

where $\mathrm{Ti}$ is the temperature at a specific point, $\mathrm{Ta}$ is the average temperature of the target material to be heated, and T0 is the initial temperature. A lower COV value implies that the target material is heated more uniformly. The power absorption efficiency (PAE, $\eta$ ), which is a value indicating the energy absorption efficiency, has also been widely employed together with COV to understand the heating phenomenon [31].

$$
\eta=\frac{P_{a}}{P_{i}}
$$

$P_{i}$ is the total energy supplied to the model, and $P_{a}$ is the absorbed power, which was calculated through the volumetric integration of the power loss density. A higher PAE value indicates that the supplied energy is more efficiently converted into heat. In this study, the positions and sizes of hot spots that occurred inside the cavity were confirmed through an electromagnetic field distribution, and the heating uniformity and efficiency were analyzed for various parameters using the COV and PAE values. 


\subsection{Effect of Cavity Height}

Because the distribution of the electromagnetic field sensitively changes depending on the geometry of the cavity, analysis was conducted according to the cavity height first. The cavity height was increased by $12.2 \mathrm{~mm}$, which corresponds to a tenth of the wavelength of the $2.45 \mathrm{GHz}$ microwave, from $461 \mathrm{~mm}$, and the total increase was $122 \mathrm{~mm}$. Figure 6 shows the electromagnetic field distribution according to height. Finding pattern of the electromagnetic field distribution according to height using the electromagnetic field distribution map alone was challenging, and so was predicting the distribution of hot spots and cold spots. For example, in the case of the cavity whose height was increased by 0.1 times the wavelength, cold spots were formed in the central part, but hot spots were formed in parts close to the surface. When the cavity height was increased by 0.5 times the wavelength, cold spots were formed toward the surface and wide hot spots were formed in the central part. This shows significant changes in the electromagnetic field despite a small change in geometry and confirms that it is almost impossible to intuitively predict the electromagnetic field distribution.
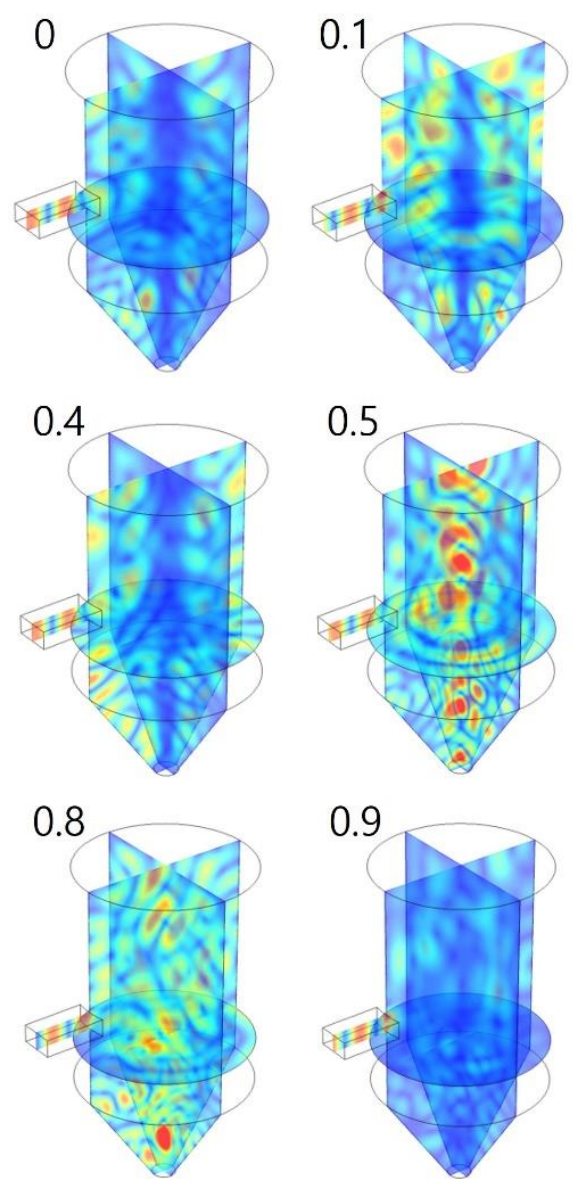
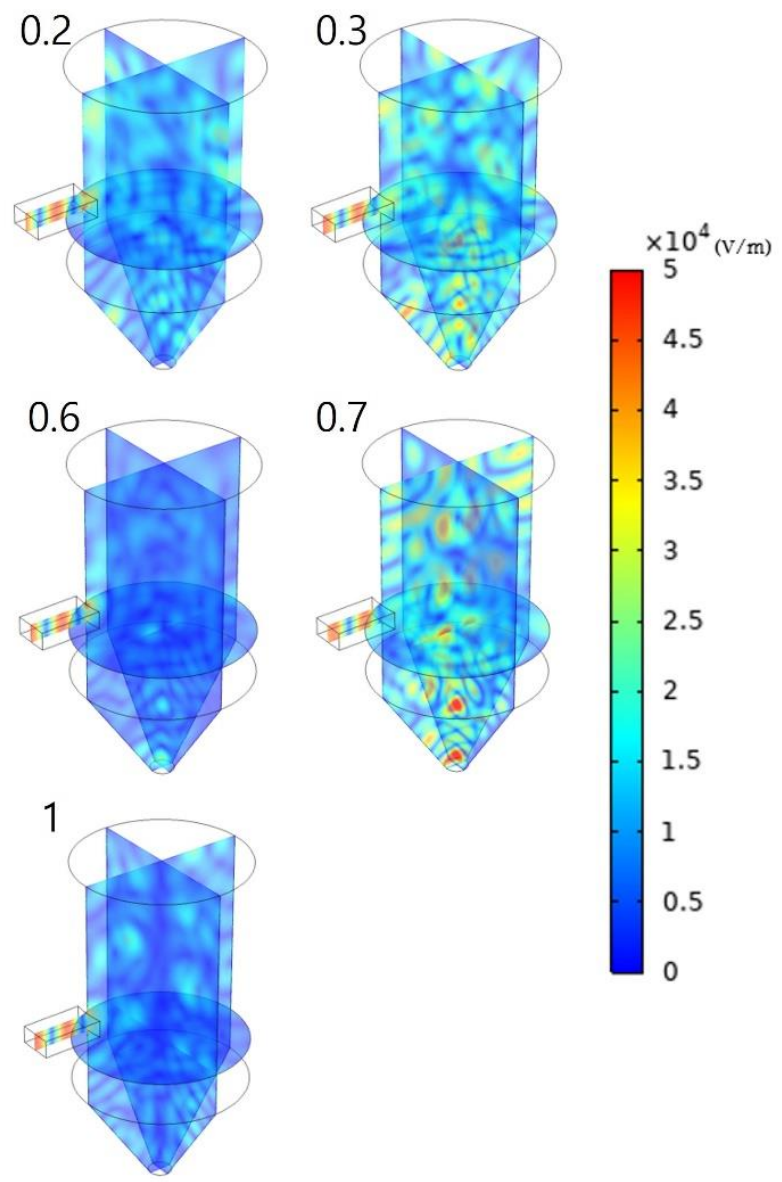

Figure 6. Electric field distribution by increasing the height of the cavity from 0 to 1 times the wavelength.

To examine the heating effect according to height, the COV and PAE are summarized in Figure 7. When the height was increased by 0.2 time the wavelength, the largest COV value of 0.852 was observed and the uniformity value became about 2 times higher than that when the height was increased by 0.8 wavelength and the lowest COV value was observed. The heating efficiency also significantly changed depending on height, similar to heating uniformity. When the height was increased by 0.5 times the wavelength, a total of $141 \mathrm{~W}$ of energy was absorbed when the PAE value was $0.141(14.1 \%)$, and the absorption rate was about 11 times higher than when the height was increased by 0.6 times the 
wavelength and the lowest PAE value was observed. A comparison between the PAE value and the electromagnetic field distribution summarized in Figure 6 reveals that the PAE value was also high when a strong electromagnetic field was generated. The positions and number of hot spots in the target material changed depending on the microwave cavity height due to changes in the electromagnetic field distribution and the amount of electromagnetic energy passing through the polycarbonate, resulting in differences in temperature uniformity and energy absorption efficiency. This indicates that the cavity height is a design parameter, which significantly affects the heating effect and uniformity.

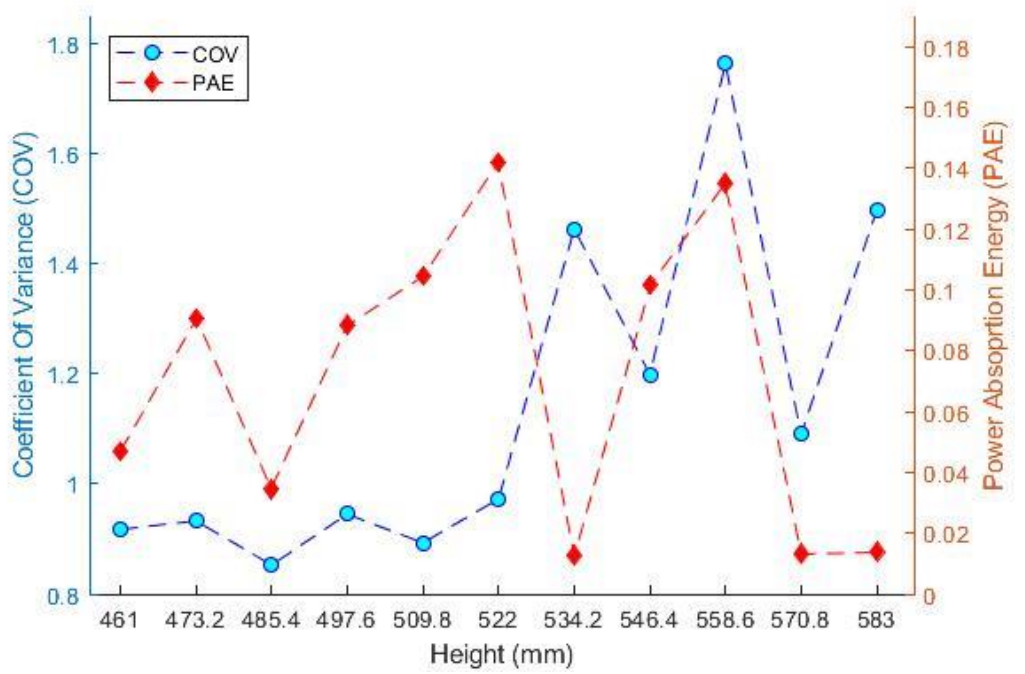

Figure 7. Coefficient of variance (COV) and power absorption efficiency (PAE) along cavity height.

\subsection{Effect of Reflector}

When an object is irradiated with microwaves, three reactions occur, that is, some waves are reflected, some pass through, and some are absorbed. Transparency and absorption are intrinsic characteristics of the material and vary depending on the wavelength of the irradiated microwaves. Reflection is related to the target material, but it mainly occurs in the cavity because the reflectivity of the target material is relatively low. Thus, the heating pattern can be adjusted by changing the geometry of the cavity because it changes the energy distribution of microwaves. In this regard, research has been widely conducted to achieve more uniform heating than before internal modification by adjusting the reflection of electromagnetic waves through a change in the cavity geometry or installing objects, such as a rotating body or a conveyor belt, inside the cavity [32]. In this study, spherical, cylindrical, and conical reflectors that reflect microwaves were installed inside the microwave cavity, and changes in the electromagnetic field and the heating effect were analyzed. To evaluate the performance of the cavity in various directions, Comprehensive evaluation coefficient (CEC), which can consider the heating efficiency and heating uniformity, as well as PAE and COV, which were introduced earlier, was introduced. CEC is defined as follows:

$$
C E C=P A E / C O V
$$

A higher CEC value indicates a higher energy absorption rate and more uniform heating. Therefore, CEC can be used as a comprehensive parameter [33].

\subsubsection{Effect of Reflector Shape}

Conical, spherical, and cylindrical reflectors were placed inside the cavity and on the opposite side of the magnetron. Their dimensions and geometry are summarized in Figure 2. To analyze the influence of the installed reflectors on the internal environment and 
heating effect, simulation was performed by applying three types of reflectors and changing their cavity heights. As can be seen from the electromagnetic field distribution in Figure 8 , more notable changes in electromagnetic field were observed when the reflectors were installed than when only the height of the cavity was changed. The highest intensity of the electromagnetic field with no height change was $4.82 \times 10^{4} \mathrm{~V} / \mathrm{m}$ for the existing model; however, it increased by approximately $60 \%$ to $7.84 \times 10^{4} \mathrm{~V} / \mathrm{m}$ when a conical reflector was installed. The spherical and cylindrical reflectors also increased the highest intensity of the electromagnetic field to $4.88 \times 10^{4}$ and $5.82 \times 10^{4} \mathrm{~V} / \mathrm{m}$, respectively.

(a)

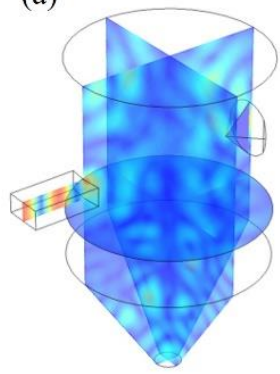

(b)

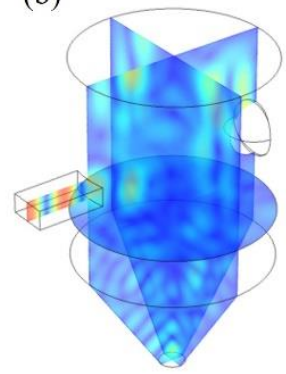

(c)

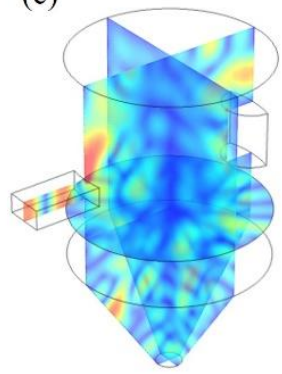

(d)

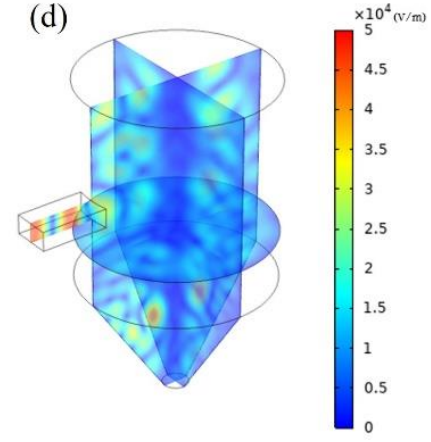

Figure 8. Electric field distribution with different reflector shape such as (a) conical (b) spherical (c) cylindrical (d) no installation.

Simulation was performed for 44 cases while the reflector geometry ( 4 cases) and the cavity height (11 cases) were varied; the COV and PAE values are summarized in Table 2. Whether more uniform heating occurs in the presence of reflectors was analyzed based on the case with no reflector. Among 33 cases with reflectors, lower COV values were observed in the presence of reflectors in 19 cases (57\%), confirming more uniform heating than in the models with no reflector. In addition, the PAE value was higher in the presence of reflectors in 21 cases (63\%) out of the 33 cases, showing that the installation of reflectors can increase the energy absorption. In particular, for the cavity whose height increased by 0.2 times the wavelength compared to the ordinary model, the energy absorption was approximately $3 \%$ when no reflector was installed, but it increased by at least twice when any reflector was installed.

Figure 9 shows the CEC values for 44 cases. For the CEC value that reflected both the energy efficiency and heating uniformity, 66\% (filled marks in Figure 9) exhibited better values than the ordinary model with no reflector. When the reflectors were compared, the spherical and conical reflectors exhibited more excellent performance than the cylindrical reflector. When only the spherical and conical reflectors were considered, 16 cases (72\%) out of 22 exhibited higher CEC values than the model with no reflectors. In particular, in the case of the spherical reflector, 9 cases (81\%) out of the 11 cases for the height showed better performance. This appears to be because the spherical and conical reflectors reflected electromagnetic waves in three dimensions, whereas the cylindrical reflector reflected them in two dimensions. The installation of reflectors was found to be an important factor for cavity design as it changed the electromagnetic field distribution more notably even though it did not always lead to more uniform and higher heating efficiency. 
Table 2. COV and PAE values with different reflector shape and height of the cavity.

\subsection{Effect of Double Waveguides}

\begin{tabular}{|c|c|c|c|c|c|}
\hline Height (mm) & Evaluation & Conical & Spherical & Cylindrical & No installation \\
\hline \multirow{2}{*}{461} & $\mathrm{COV}$ & 0.638 & 1.349 & 0.612 & 0.917 \\
\hline & PAE & 0.033 & 0.016 & 0.079 & 0.047 \\
\hline \multirow{2}{*}{473.2} & $\mathrm{COV}$ & 1.861 & 2.010 & 1.138 & 0.933 \\
\hline & PAE & 0.271 & 0.348 & 0.058 & 0.091 \\
\hline \multirow{2}{*}{485.4} & $\mathrm{COV}$ & 0.646 & 0.856 & 0.640 & 0.853 \\
\hline & PAE & 0.067 & 0.384 & 0.233 & 0.034 \\
\hline \multirow{2}{*}{497.6} & $\mathrm{COV}$ & 0.690 & 0.979 & 1.095 & 0.945 \\
\hline & PAE & 0.087 & 0.145 & 0.074 & 0.089 \\
\hline \multirow{2}{*}{509.8} & $\mathrm{COV}$ & 1.549 & 1.773 & 1.240 & 0.893 \\
\hline & PAE & 0.059 & 0.060 & 0.021 & 0.105 \\
\hline \multirow{2}{*}{522} & $\mathrm{COV}$ & 0.982 & 0.795 & 0.898 & 0.973 \\
\hline & PAE & 0.098 & 0.135 & 0.050 & 0.142 \\
\hline \multirow{2}{*}{534.2} & $\mathrm{COV}$ & 1.607 & 0.800 & 1.183 & 1.463 \\
\hline & PAE & 0.013 & 0.043 & 0.113 & 0.013 \\
\hline \multirow{2}{*}{546.4} & $\mathrm{COV}$ & 1.090 & 0.582 & 0.683 & 1.199 \\
\hline & PAE & 0.205 & 0.168 & 0.113 & 0.102 \\
\hline \multirow{2}{*}{558.6} & $\mathrm{COV}$ & 1.575 & 0.978 & 0.894 & 1.764 \\
\hline & PAE & 0.042 & 0.164 & 0.163 & 0.135 \\
\hline \multirow{2}{*}{570.8} & $\mathrm{COV}$ & 0.800 & 0.738 & 1.218 & 1.091 \\
\hline & PAE & 0.034 & 0.019 & 0.015 & 0.013 \\
\hline \multirow{2}{*}{583} & $\mathrm{COV}$ & 1.244 & 0.679 & 1.650 & 1.497 \\
\hline & PAE & 0.0401 & 0.093 & 0.033 & 0.014 \\
\hline
\end{tabular}

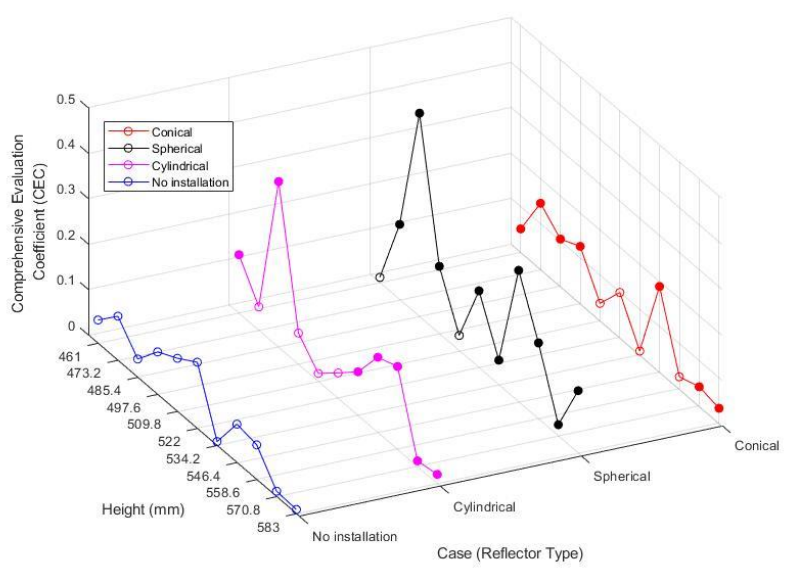

Figure 1. Comprehensive evaluation coefficient (CEC) with different reflector shape and height of the cavity.

In the preceding chapters, changes in electromagnetic field and heat distribution due to changes in cavity geometry were analyzed. Changing the cavity geometry and the method of using multiple waveguides has also been proposed as measures to achieve uniform and high-efficiency heating [21]. Most studies, however, focused on the application of multiple waveguides to the fixed cavity, but studies on the influence of detailed cavity design parameters, such as the position, port direction, and power, on the electromagnetic field distribution and heating effect, are insufficient and remain on a laboratory scale. Therefore, waveguides were additionally installed in the cavity of various geometries, and 
the temperature uniformity and energy efficiency of the target material during heating were analyzed.

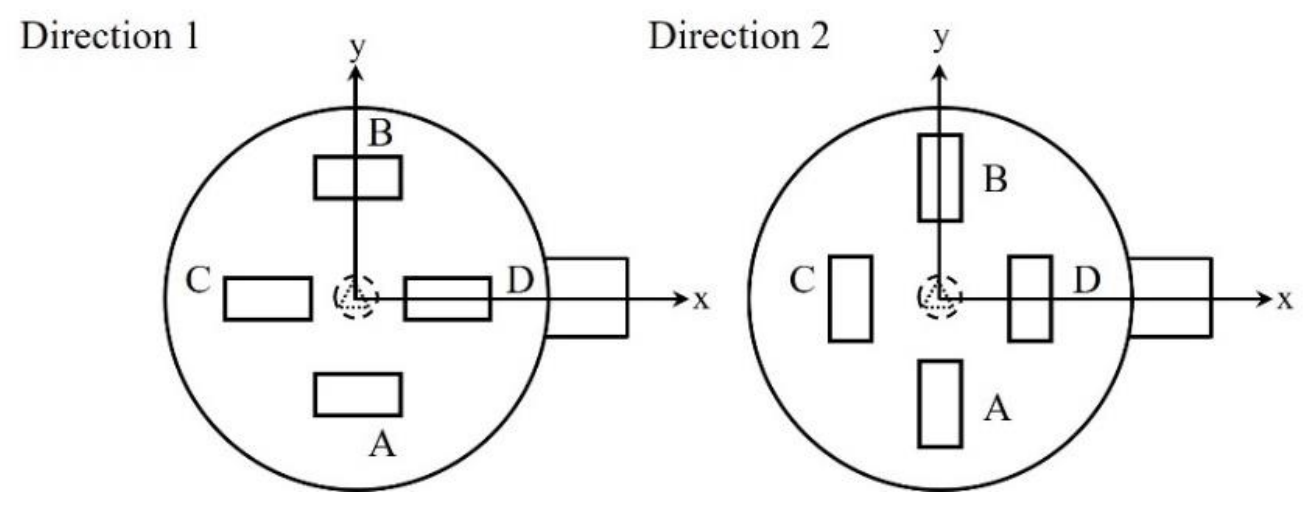

Figure 2. Schematic illustration presenting the position of additional waveguide.

\subsubsection{Effect of Double Waveguides Position and Direction}

To analyze the influence of the double waveguides, another waveguide was installed in the upper part in addition to the existing waveguide position. The waveguides were vertically arranged on the side and in the upper part of the cavity to reduce the resonance and offset between the electromagnetic waves from them. Figure 10 summarizes the geometry and installation positions of the waveguides. In the case of the waveguide placed in the upper part, eight cases were simulated based on four positions and two directions. The two waveguides had dimensions of WR-340, and each waveguide had a power of 0.5 $\mathrm{kW}$ so that a total of $1 \mathrm{~kW}$ of energy could be emitted as in the single waveguide model.

As shown in the surface electromagnetic field distribution in Figure 11, a hot spot occurred at the top center of the target material when double waveguides were installed, and it was wider compared to the existing single waveguide model. When double waveguides were installed, the intensity of the electromagnetic field was found to be higher than the maximum intensity of the electromagnetic field in the existing model $\left(2.54 \times 10^{4}\right.$ $\mathrm{V} / \mathrm{m})$, irrespective of the parameters.

\section{Direction 1}
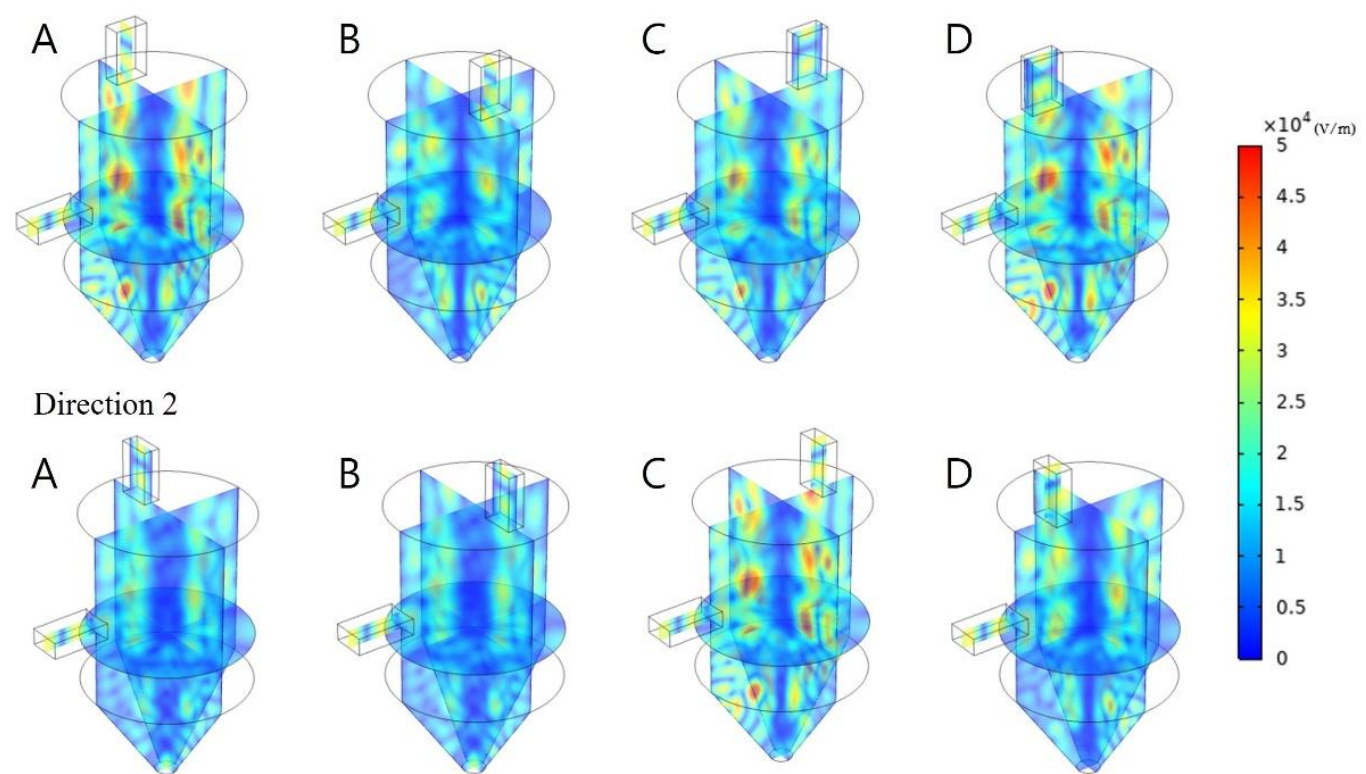

Direction 2
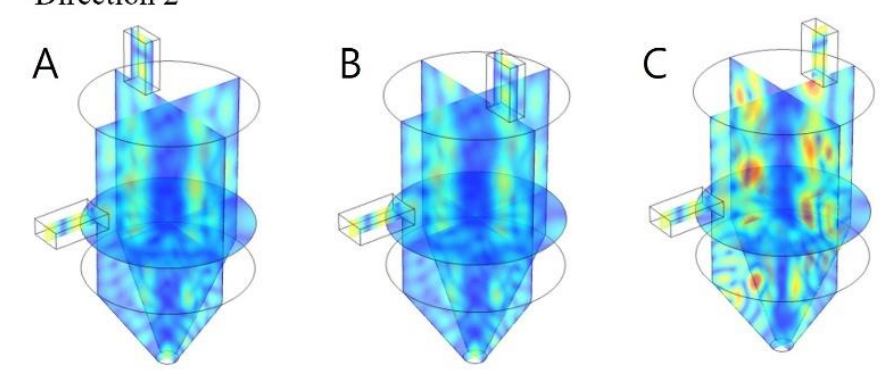

Figure 3 Electric field distribution with different reflector position and direction under $0.5 \mathrm{~kW}$ irradiation each. 
Table 3 shows the COV and PAE for the eight positions and directions of the waveguides. First, in the case of Direction 1, the most uniform heating was observed when the waveguide was located at position D in Figure 11, where the COV value was found to be 0.659. All four positions were more uniformly heated than when the single waveguide was used, and the average COV value was 0.710 , exhibiting $22 \%$ higher uniformity than when one waveguide port was used. The highest energy absorption was also observed at position D similar to the COV, with the PAE value being 0.116 . The PAE value was also higher at all positions than when the single waveguide was used. The average value of the four positions was 0.08 , which was $22 \%$ higher than that of the ordinary model.

In the case of Direction 2, when the waveguide was located at position D, the most uniform heating was achieved among eight cases as the COV value was 0.566 . The average COV value for the four positions was 0.701, which was higher than that when the single waveguide was installed. The average PAE value for the four positions was also 0.059, which was excellent compared to that when the single waveguide was installed.

Consequently, when double waveguides were used, more excellent heating uniformity and energy absorption were observed in all eight cases. The use of the double waveguides made it possible to reach area that were difficult to reach when microwaves were irradiated from the side by irradiating microwaves in the vertical direction as well, thereby causing more uniform heating. The target material was uniformly heated, and the energy absorption increased compared to when the single waveguide was used because the cases of generating hot spots due to the concentration of electromagnetic waves caused by interference and cold spots due to the offset between the microwaves decreased.

Table 3. Coefficient of variance (COV) and power absorption efficiency (PAE)with different reflector position and direction under 0.5 $\mathrm{kW}$ irradiation each.

\begin{tabular}{ccccccccc}
\hline \multirow{2}{*}{ Direction } & Evaluation & A & B & C & D & $\begin{array}{c}\text { Single } \\
\text { Average } \\
\text { Waveguide }\end{array}$ \\
\hline \multirow{2}{*}{1} & COV & 0.686 & 0.780 & 0.717 & 0.659 & 0.710 & 0.917 \\
\cline { 2 - 8 } & PAE & 0.081 & 0.052 & 0.080 & 0.116 & 0.0823 & 0.046 \\
\hline \multirow{2}{*}{2} & COV & 0.830 & 0.831 & 0.578 & 0.566 & 0.701 & 0.917 \\
\cline { 2 - 8 } & PAE & 0.050 & 0.050 & 0.087 & 0.049 & 0.059 & 0.046 \\
\hline
\end{tabular}

3.3.2 Effect of Double Waveguides Power

In the preceding section, the effect of double waveguides was confirmed. For their application in industries, the heating effect according to supplied power is also an important element. Therefore, in this section, the heating effect was compared and analyzed while the power per waveguide was increased from 0.5 to $1 \mathrm{~kW}$. The electromagnetic field distribution map in Figure 12 shows that when the power of the waveguides was increased, the distribution of the electromagnetic field remained similar, but its intensity increased. Thus, the position of the hot spot also remained similar, but its size increased.

When the COV and PAE values described in Table 4 were calculated, the COV value was found to decrease regardless of the position and direction as the power per port increased from 0.5 to $1 \mathrm{~kW}$. In particular, when the power was $1 \mathrm{~kW}$ in direction 1 , the average COV value for the four positions was 0.647 , which was $117 \%$ lower than when the power was $0.5 \mathrm{~kW}$. The energy efficiency remained similar as the input power doubled even though the energy absorption almost doubled.

Because the inside of the cavity was insulated, the average temperature also doubled as the energy supply doubled. As the positions of the waveguides remained unchanged, the pattern of the temperature profile was maintained and only its intensity increased. The increased average temperature led to a relatively uniform temperature distribution because the absorbed heat could not be transferred to the outside of the cavity. This indicates that the boundary conditions as well as the cavity geometry are important design 
parameters for the cavity. The excellent insulation effect of the cavity can save energy and create a uniform temperature distribution by preventing the loss of thermal energy.

Direction 1

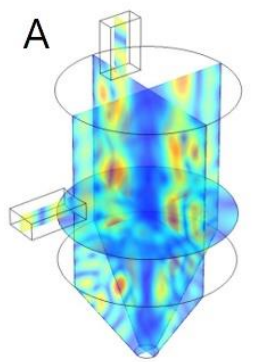

Direction 2

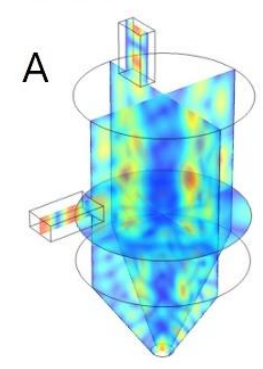

B

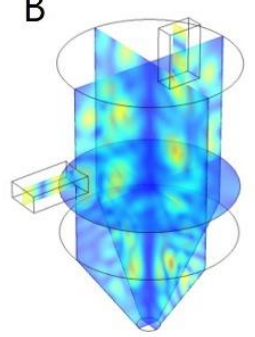

B

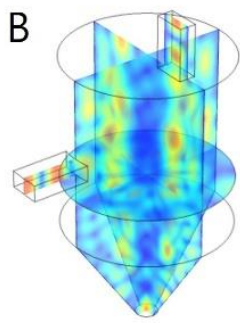

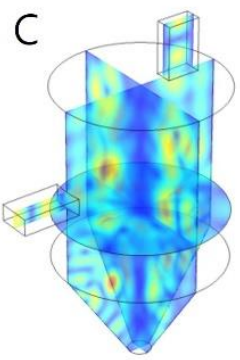

C

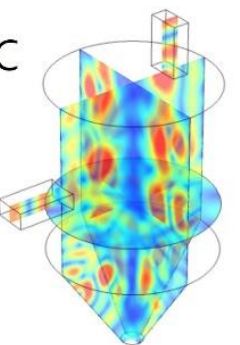

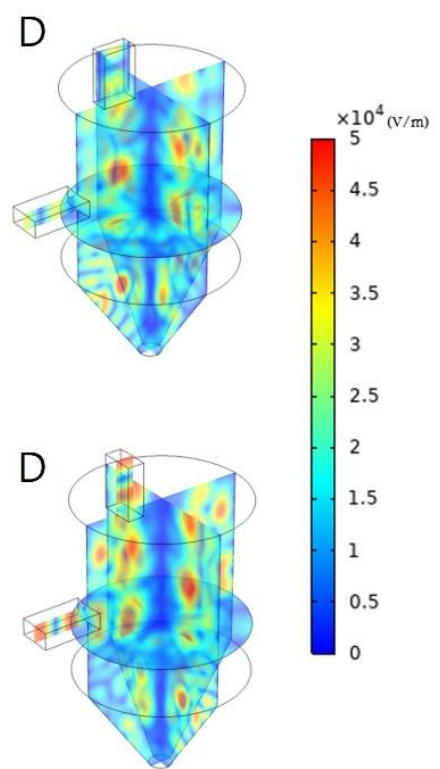

Figure 4. Electric field distribution with different reflector position and direction under $1 \mathrm{~kW}$ irradiation each.

Table 4. Coefficient of variance (COV) and power absorption efficiency (PAE) with different reflector position and direction under 1 $\mathrm{kW}$ irradiation each.

\begin{tabular}{ccccccc}
\hline Direction & Evaluation & A & B & C & D & Average \\
\hline \multirow{2}{*}{1} & COV & 0.631 & 0.666 & 0.663 & 0.628 & 0.647 \\
\cline { 2 - 7 } & PAE & 0.081 & 0.052 & 0.080 & 0.116 & 0.082 \\
\hline \multirow{2}{*}{2} & COV & 0.827 & 0.829 & 0.576 & 0.565 & 0.699 \\
\cline { 2 - 7 } & PAE & 0.050 & 0.050 & 0.087 & 0.049 & 0.059 \\
\hline
\end{tabular}

\section{Conclusion}

In this study, the microwave heating effect was analyzed on a pilot scale according to the design parameters, such as the cavity height, the installation of reflector and type, the number and positions of waveguides. The electromagnetic field and temperature were calculated using finite element analysis considering electromagnetic and thermal effect, and were compared with the experimental results. The following conclusions could be drawn:

(1) Despite a slight change in the cavity height caused by changing the wavelength by one-tenth, the electromagnetic field distribution and parameters, such as the COV and PAE, were sensitively changed.

(2) The installation of reflectors significantly changed the electromagnetic field. When reflectors were installed, uniform heating was generally achieved, and the power absorption also increased. An evaluation of the CEC, a comprehensive coefficient that reflects both PAE and COV, confirmed that the spherical reflector has the highest efficiency.

(3) When double waveguides were used, the target material was uniformly heated, and the energy absorption increased at the same time because electromagnetic waves reached area that were difficult to reach using the single waveguide and the interference phenomenon was reduced by the vertical arrangement of waveguides. 
(4) As the applied power increased, the form of the electromagnetic field distribution did not change but the size of the hot spot increased. The heat inside the cavity increased due to the increased power supply, and the increase in the average temperature of the target material led to uniform heating.

The above observations revealed that the cavity geometry, the application of reflector, the arrangement of waveguides, and the insulation conditions of the cavity are important parameters for cavity design. Furthermore, this study will be helpful in applying microwaves to actual industrial sites because it deals with the pilot scale instead of the lab scale, which has been mainly researched. It is expected that microwaves will be applied to product processing industries, such as synthesis, decomposition, and phase changes, beyond material heating.

Author Contributions: D.J. and S.J. conceived and designed the experiments; S.J. and J.K. performed the experiments; D.J. and S.J. analyzed the data; S.J. and J.K. evaluated validation D.J. and S.J. wrote the article; D.J., S.J. and J.K. reviewed article. All authors have read and agreed to the published version of the manuscript.

Funding: This research was supported by the Human Resource Training Program(S2755803) for business-related research and development of Ministry of SMEs and Startups in 2019.

Institutional Review Board Statement: Not applicable.

Informed Consent Statement: Not applicable.

Data Availability Statement: Data available in a publicly accessible repository

Conflicts of Interest: The authors declare no conflict of interest.

\section{References}

1. International Energy Agency (EIA) : https://www.iea.org/articles/global-energy-review-co2-emissions-in-2020

2. Nielsen, T.D, Hasselbalch, J., Holmberg, K., Stripple, J., Politics and the plastic crisis a review throughout the plastic life cycle. Wires Energy And Environment 2020, 9, 154-196.

3. Oyeyinka, S. A., Akintayo, O. A., Adebo, O. A., Kayitesi, E., Njobeh, P. B., A review on the physicochemical properties of starches modified by microwave alone and in combination with other methods. International Journal of Biological Macromolecules 2021, 176, 87-95.

4. Wei, W., Shao, Z.S., Chen, W.W., Zhang, P.J., Yuan, Y., A Fully Coupled Electromagnetic Irradiation, Heat and mass transfer model of microwave heating on concrete., IEEE Access 2020, 9, 1575-1589.

5. Zhu, H., He, J., Hong, T., Yang, Q., Wu, Y., Yang, Y., Huang, K., A rotary radiation structure for microwave heating uniformity improvement., Applied Thermal Engineering 2018, 141, 648-658.

6. Zhan, L., Yang, Y., Wan, L., Wang, G., Wang, N., Drying kinetics and mechanical properties of low temperature microwave dried cashmere fibers., Textile Research Journal 2020, 90, 2745-2754.

7. Amini, A., Latifi, M., \& Chaouki, J., Electrification of materials processing via microwave irradiation: A review of mechanism and applications. Applied Thermal Engineering 2021, 193, 117003.

8. Jie, X., Li, W., Slocombe, D., Gao, Y., Banerjee, I., Gonzalez-Cortes, S., Yao, B., Almegrem, H., Alshihri, S., Dilworth, J., Xiao, T., Edwards, P., Microwave-initiated catalytic deconstruction of plastic waste into hydrogen and high-value carbons. Nature Catalysis 2020, 3, 902-912.

9. Suriapparao, D. V., Vinu, R., Biomass waste conversion into value-added products via microwave-assisted Co-Pyrolysis platform. Renewable Energy 2021, 170, 400-409.

10. Li, Q., Ling, Y., Zheng, H., Chen, G., Chen, J., Koppala, S., Jiang, Q., Li, K., Omran, M., Gao, L., Phase microstructure and morphology evolution of MgO-PSZ ceramics during the microwave sintering process. Ceramics International 2021, 47, 1584915858.

11. Gupta, D., Sharma, A. K., Microwave cladding: a new approach in surface engineering. Journal of Manufacturing Processes 2014, $16,176-182$.

12. Aguilar-Reynosa, A., Romani, A., Rodriguez-Jasso, R. M., Aguilar, C. N., Garrote, G., Ruiz, H. A., Microwave heating processing as alternative of pretreatment in second-generation biorefinery: An overview. Energy Conversion and Management 2017, 136, 5065.

13. Farag, S., Sobhy, A., Akyel, C., Doucet, J., Chaouki, J., Temperature profile prediction within selected materials heated by microwaves at 2.45 GHz. Applied Thermal Engineering 2012, 36, 360-369.

14. Li, Z. Y., Wang, R. F., Kudra, T. J. D. T., Uniformity issue in microwave drying. Drying Technology 2011, 29, 652-660. 
15. Yi, Q., Lan, J., Ye, J., Zhu, H., Yang, Y., Wu, Y., Huang, K., A simulation method of coupled model for a microwave heating process with multiple moving elements. Chemical Engineering Science 2021, 231, 116339.

16. He, J., Yang, Y., Zhu, H., Li, K., Yao, W., Huang, K., Microwave heating based on two rotary waveguides to improve efficiency and uniformity by gradient descent method. Applied Thermal Engineering 2020, 178, 115594.

17. Tang, Z., Zhang, S., Hong, T., Zhu, H., \& Huang, K. (2018, May). Improvement of Microwave Heating Performance via a New Combination Method. In 2018 International Conference on Microwave and Millimeter Wave Technology (ICMMT) (pp. 1-3). IEEE. (Conference)

18. Halim, S. A., Swithenbank, J., Simulation study of parameters influencing microwave heating of biomass. Journal of the Energy Institute 2019, 92, 1191-1212.

19. Chen, J., Lau, S. K., Chen, L., Wang, S., Subbiah, J., Modeling radio frequency heating of food moving on a conveyor belt. Food and Bioproducts Processing 2017, 102, 307-319.

20. Zhang, R., Wang, Y., Wang, X., Luan, D., Study of heating characteristics for a continuous $915 \mathrm{MHz}$ pilot scale microwave thawing system. Food Control 2019, 104, 105-114.

21. Mokhta, Z. M., Ong, M. Y., Salman, B., Nomanbhay, S., Salleh, S. F., Chew, K. W., Chew, K.W., Chen, W. H., Simulation studies on microwave-assisted pyrolysis of biomass for bioenergy production with special attention on waveguide number and location. Energy 2020, 190, 116474.

22. Li, H., Shi, S., Lin, B., Lu, J., Lu, Y., Ye, Q., Wang, Z., Hong, Y., Zhu, X., A fully coupled electromagnetic, heat transfer and multiphase porous media model for microwave heating of coal. Fuel Processing Technology 2019, 189, 49-61.

23. COMSOL, 2021. Heat Transfer Module User's Guide. COMSOL Multiphysics $®$ V5.6: www.comsol.com.

24. Amini, A., Ohno, K., Maeda, T., Kunitomo, K., Effect of particle size and apparent density on the initial stages of temperature increase during the microwave heating of Fe3O4. Powder Technology 2018, 338, 101-109.

25. Buttress, A., Jones, A., \& Kingman, S., Microwave processing of cement and concrete materials-towards an industrial reality? Cement and Concrete Research 2015, 68, 112-123.

26. Lin, B., Li, H., Chen, Z., Zheng, C., Hong, Y., Wang, Z. Sensitivity analysis on the microwave heating of coal: A coupled electromagnetic and heat transfer model. Applied Thermal Engineering 2017, 126, 949-962

27. Tamang, S., Aravindan, S., 3D numerical modelling of microwave heating of SiC susceptor. Applied Thermal Engineering 2019, 162, 114250.

28. Cui, K., Liao, T., Qiu, C., Chen, H., Zhou, J., Microwave-induced heating behavior of Y-TZP ceramics under multiphysics system. Green Processing and Synthesis 2020, 9, 119-130.

29. Wu, Y., Yan, B., Yang, Y., Zhu, H., Huang, K., Accordion microwave oven for uniformity and efficiency heating. International Journal of RF and Microwave Computer-Aided Engineering 2020, 30, e22190.

30. Gao, X., Liu, X., Yan, P., Li, X., \& Li, H., Numerical analysis and optimization of the microwave inductive heating performance of water film. International Journal of Heat and Mass Transfer 2019, 139, 17-30.

31. Zhou, J., Yang, X., Chu, Y., Li, X., \& Yuan, J., A novel algorithm approach for rapid simulated microwave heating of food moving on a conveyor belt. Journal of Food Engineering 2020, 282, 110029.

32. Ye, J., Xia, Y., Yi, Q., Zhu, H., Yang, Y., Huang, K., Shi, K., Multiphysics modeling of microwave heating of solid samples in rotary lifting motion in a rectangular multi-mode cavity. Innovative Food Science \& Emerging Technologies 2021, 73, 102767.

33. Zhang, Z., Su, T., Zhang, S., Shape effect on the temperature field during microwave heating process. Journal of Food Quality, 2018, 2018, 9169875. 